\title{
HYPOTHESIS
}

\section{Increased capillary permeability in systemic sclerosis: help or hindrance?}

\author{
Walter Grassi, Paolo Core, Claudio Cervini
}

\section{Department of Rheumatology, \\ University of Ancona, Italy \\ W Grassi \\ P Core \\ C Cervini}

Correspondence to: Dr Walter Grassi, Clinica Reumatologica, Ospedale 'Murri', via dei Colli 52, 60035 Jesi (An), Italy.

Accepted for publication 14 March 1996.

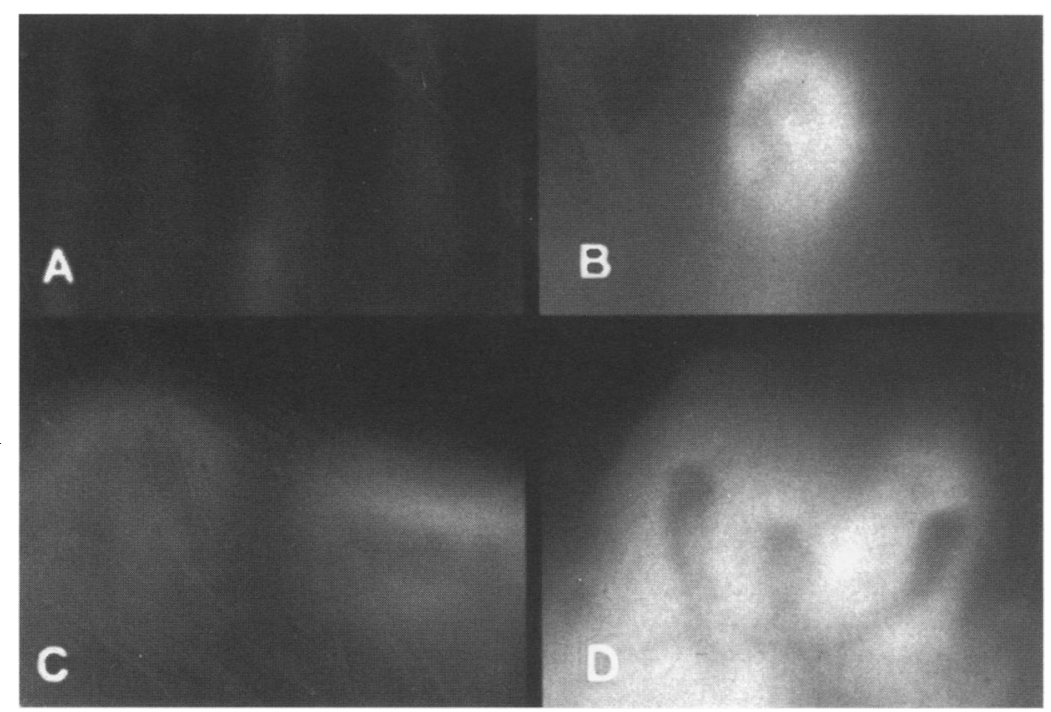

Figure 1 Fluorescence videomicroscopy. A: Homogeneous pericapillary distribution of sodium fluorescein in a control subject. B: Increased inhomogeneous transcapillary diffusion of sodium fluorescein in a normal shaped loop in a patient with systemic sclerosis (SSc). C: Marked increase in capillary permeability along the whole profile of a megacapillary in a patient with SSc. D: Definite loss of diffusion barrier around a cluster of newly formed capillaries in a patient with SSc. and tissue plasminogen activator ${ }^{10}$ in patients with SSc. Distinctive microvascular changes in SSc include: dilated and distorted capillary loops, ${ }^{11}$ diminished capillary density often culminating in regional avascularity, ${ }^{12}$ prolonged phases of reduced or ceased capillary perfusion as a result of cold induced peripheral vasospasm, and partial occlusion of the capillary lumen by platelet aggregates. ${ }^{13-15}$ Moreover, it has been well documented that capillary permeability to small solutes such as sodium fluorescein is increased and more rapid
Systemic sclerosis (SSc) is a connective tissue disease characterised by an increased secretion of normal extracellular matrix proteins in mesenchymal tissue, and by widespread morphofunctional capillary abnormalities. ${ }^{12}$ Most of the clinical features of SSc are clearly related to vascular lesions; moreover, a diffuse microangiopathy is a prominent feature in all tissues, regardless of any overt clinical involvement. ${ }^{3-5}$ Diffuse endothelial damage caused by a cytotoxic factor could be the initial lesion ${ }^{6}$ : such damage has been clearly demonstrated by ultrastructural studies ${ }^{8}$ and by the finding of increased plasma von Willebrand's activity, factor VIII/von Willebrand's factor antigen,

\section{Current views of mechanisms}

Although the pathophysiology of the increased capillary permeability in SSc is not clearly understood, microvascular lesions are considexed a crucial pathogenetic factor in determining the less effective diffusion barrier. It has been hypothesised that widespread endothelial cell damage may be the precursor of small vessel disease, and could lead to increased vascular permeability ${ }^{13}$ and the subsequent development of fibrosis. ${ }^{22}$ The increased capillary permeability assessed by dynamic fluorescence videomicroscopy has been regarded as the consequence of capillary wall damage, ${ }^{16}$ and a link between endothelial injury and increased capillary permeability has also been proposed to explain the pathophysiological mechanisms of vascular disease in diabetes mellitus. ${ }^{23}$ However, there are no compelling experimental data demonstrating that increased capillary permeability has a clearly defined pathogenetic role in SSc.

\section{Contradictions}

If the increased capillary permeability in SSc is to be regarded as the pathological consequence 
of a damaged diffusion barrier of the capillary loops, and if we accept that it can contribute to the development of fibrosis, ${ }^{22}$ then it would be reasonable to antagonise such an increase in permeability and to avoid any sort of influence that could further increase both the transcapillary diffusion and the interstitial distribution of plasma solutes. This is contradicted by two main observations. First, cold induced vasospasm, that causes a dramatic decrease both in peripheral perfusion ${ }^{5024}$ and in the flow related capillary permeability, ${ }^{25}$ has well known negative effects ${ }^{26-28}$ on the disease course; and second, calcium channel blockers, that induce an increase of both capillary flow and permeability, ${ }^{29}$ are helpful in treating patients with SSc. ${ }^{30-33}$

\section{Hypothesis}

Various observations support the intriguing hypothesis that the increased capillary permeability in SSc could represent a physiological protective mechanism in areas with critical nutritional exchanges.

It has been demonstrated that capillary permeability increases when critical hypoxia occurs. ${ }^{34}$ In patients with SSc there occur several abnormalities capable of inducing tissue hypoxia. These include loss of capillaries, ${ }^{35}$ decreased diffusion coefficient of oxygen as a result of fibrosis ${ }^{34}$ decreased oxygen supply in the peripheral diffusion zone as a result of both increase in the thickness of vascular layers ${ }^{36} 37$ and fibrin deposition in the perivascular tissues $^{38}$ enhanced neutrophil and platelet adherence to damaged endothelium, altered microcirculatory hydrostatics, and impaired vasomotion. ${ }^{14}$ Loss of capillaries, in particular, could be relevant in determining critical tissue hypoxia. A decreased capillary density is an early typical feature of SSc, ${ }^{12}$ and it has been estimated that the number of capillaries may be reduced to perhaps only $20 \%$ of normal. ${ }^{22}$ As a consequence, each single capillary loop would be required to serve for nutritional exchanges over a wider interstitial domain than in normal conditions.

In these dramatic conditions of microvascular impairment in patients with SSc, microvascular dynamics are further worsened by cold induced vasospasm, as demonstrated by the significant decrease in pericapillary and interstitial diffusion of sodium fluorescein in patients with SSc after exposure to cold..$^{25}$ Although this decrease occurred within two minutes after the first appearance of dye at the selected capillary loops, the cold induced decrease in capillary permeability should not be assumed to be a temporary phenomenon restricted to the period of vasospasm. The intravenous bolus injection of a single small quantity of sodium fluorescein affords only a quick glimpse of the complex dynamic phenomenon of cold induced changes in capillary permeability - akin to looking into a dark room by the light of a single match.

To test the hypothesis that cold induced decrease in capillary permeability is not a temporary phenomenon, we performed a bolus injection of sodium fluorescein 15 minutes after immersion of the hand in cold water, under the same experimental conditions as used in the earlier experiments. ${ }^{25}$ We observed a marked decrease in fluorescence light intensity with respect to the baseline value (fig 2), suggesting that a dramatic decrease in capillary permeability is a long lasting phenomenon following cold induced vasospasm.

All these findings support the hypothesis that an increased baseline capillary permeability in SSc could be regarded as an adaptive physiological response to chronic tissue ischaemia caused by both the reduced capillary density and the episodic phases of cold induced low capillary perfusion. This view is further supported by the findings of Franzeck et $a l^{39}$ who demonstrated a diffuse leakage of dye from the capillaries into the interstitial space in patients with various conditions characterised by digitial artery occlusion and severely ischaemic areas.

Additional potential support for the concept that increased capillary permeability in SSc affords nutritional protection arises from the relationship between capillary dilatation and permeability. It has been reported that microvascular dilatation represents a local autoregulatory response to tissue hypoxia. ${ }^{23}$ As a dramatic increase in capillary permeability is a prominent characteristic of dilated loops and megacapillaries in patients with SSc, ${ }^{16}$ it seems reasonable to consider the resulting increased transcapillary diffusion of solutes as an effective means of achieving better nutritional exchange. It is interesting that the triad 'tissue hypoxia-enlarged loops-increased capillary
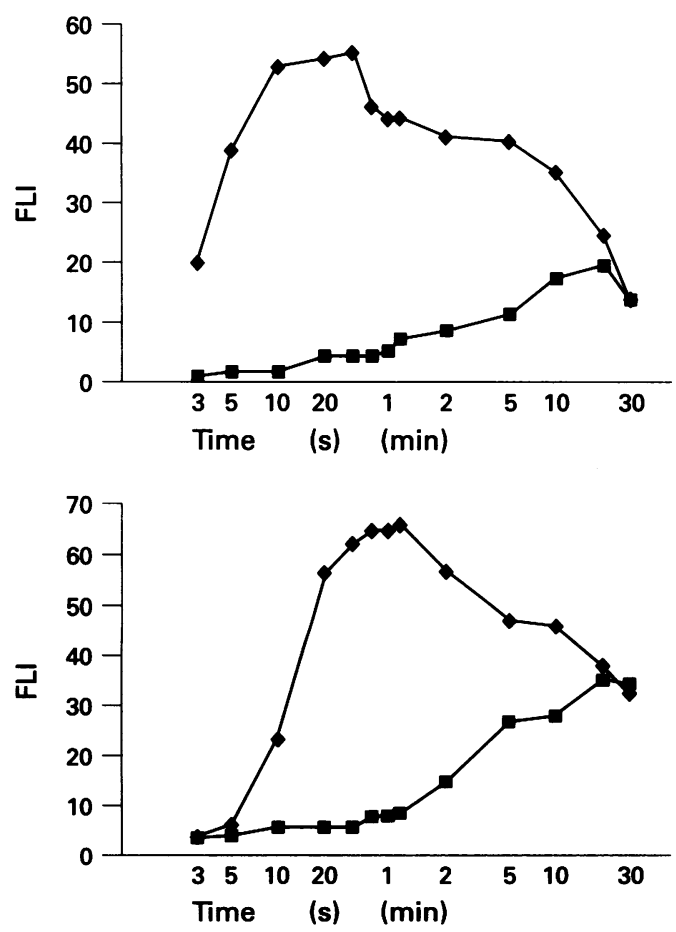

Figure 2 Fluorescent light intensity (FLI) measured at different times after the first appearance of sodium fluorescein in selected capillary loops of two patients with systemic sclerosis. Capillary permeability was assessed 15 minutes after peripheral cold exposure (hands covered with plastic gloves and immersed for three minutes in water at plastic gloves and immersed for three minutes in
$\left.5^{\circ} \mathrm{C}\right) . \quad=$ Basal condition; $\square=$ after cold test. 
permeability' occurs both in SSc and in diabetes mellitus ${ }^{41}{ }^{42}$ : transcapillary and interstitial diffusion of sodium fluorescein is increased in diabetes mellitus that is characterised by local tissue hypoxia as a result of impaired oxygen transport. ${ }^{23} 40$

\section{Testing the hypothesis}

Experimental support for our hypothesis comes from our previous studies. We have demonstrated that nifedipine significantly antagonises the cold induced decrease in permeability and interstitial concentration of sodium fluorescein in patients with SSc. ${ }^{29}$ If it can be assumed that the significant baseline increase in capillary permeability has some pathogenetic role, then the further increase in transcapillary diffusion of solutes induced by nifedipine may be expected to worsen the disease course in patients with SSc. On the contrary, however, it is well known that calcium channel blockers have several valuable effects-antivasospastic activity, ${ }^{30}{ }^{43}$ marked improvement in unresponsive skin ulceration, ${ }^{44} 45$ regression of calcinosis, ${ }^{31}$ and improvement in the diffusing capacity of the lung. ${ }^{33}$ We are currently investigating the influence of nifedipine on capillary permeability. Our preliminary data indicate that the drug induces a significant increase in capillary permeability compared with baseline in patients with SSc (figs 3, 4).

The hypothesis of a protecting role of increased capillary permeability has also been explored by studying the effect of peripheral exposure to warmth on the transcapillary diffusion of sodium fluorescein. It has been reported that placing the hands in warm water can increase resting peripheral perfusion and reduce the reactivity to cold, ${ }^{46}$ and 'keeping warm' is a cornerstone of management of patients with Raynaud's phenomenon in SSc. ${ }^{47}$ We have found in preliminary trials that placing the hands in warm water $\left(42^{\circ} \mathrm{C}\right)$ for 10 minutes induces a significant increase in capillary permeability in patients with SSc (fig 5).

While it is necessary to be careful not to overgeneralise our conclusions, these findings

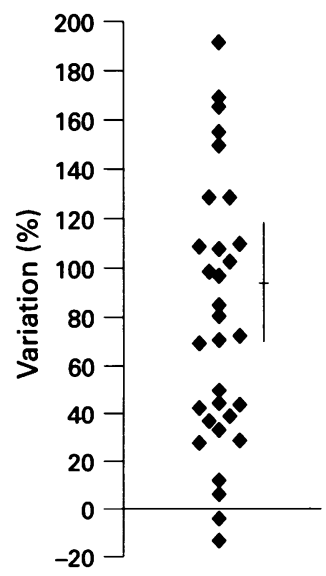

Figure 3 Scatterplot showing percent variation in fluorescent light intensity induced by nifedipine, with respect to baseline values. Data from six patients in whom a mean 4.5 loops (range 3-7) were examined in the two experimental conditions.

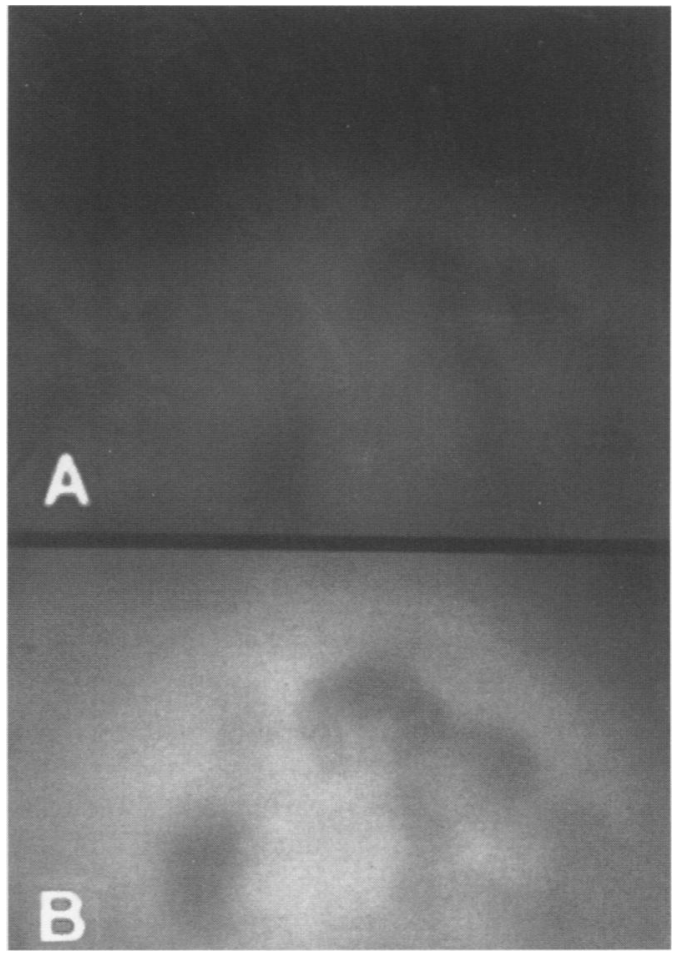

Figure 4 Representative patterns of pericapillary diffusion and interstitial distribution of sodium fluorescein in $a$ patient with systemic sclerosis. A: Baseline. B: After single oral administration of $10 \mathrm{mg}$ of nifedipine 30 minutes before the examination. Both photographs were taken one minute after the first appearance of the dye at the nail fold.

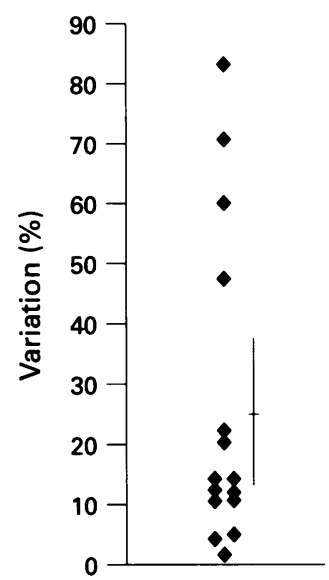

Figure 5 Scatterplot showing percent variation in fluorescent light intensity induced by exposure to warmth, with respect to baseline values. Data from three patients in whom a mean 5 loops (range 4-6) were examined in the two experimental conditions.

offer substantial support to the hypothesis that increased capillary permeability is more of a help than a hindrance in patients with SSc.

\section{Conclusion}

Microvascular involvement is a predominant finding in SSc that undoubtedly contributes to the pathogenesis of the disease. ${ }^{22}$ One expression of such involvement is the dramatic decrease in capillary density. The consequent widespread and persistent critical tissue hypoxia could be further worsened by several additional mechanisms, including episodes of cold or stress induced vasospasm. Another typical feature of the microangiopathy in SSc is the increased transcapillary and interstitial diffusion of low molecular weight tracers such as 
sodium fluorescein. Current theories indicate that this finding is a consequence of a disrupted physiological barrier as a result of capillary wall damage, but the underlying cause of the mechanisms that determine the increased permeability remains unclear. Although the altered pattern of diffusion could be related to the indurative oedema, no study has demonstrated unequivocally that the increased capillary permeability had a clearly defined role in the pathophysiology of SSc.

Our hypothesis is that the increased baseline capillary permeability in SSc might be regarded as a protective mechanism that allows enhanced nutritive function by single capillary loops to meet the demands resulting from the morphofunctional changes in the microcirculation (overall decrease in capillary density, cold induced peripheral hypoperfusion). This hypothesis does not exclude the possibility of some negative effects of the increased capillary permeability, but these could be regarded as side effects of a protective mechanism.

1 Black C M. The aetiopathogenesis of systemic sclerosis. $\mathfrak{f}$ Intern Med 1993; 234: 3-8.

2 LeRoy C E, Black C M, Fleischmajer R, et al. Scleroderma (systemic sclerosis): classification, subsets and pathogenesis. F R heumatol 1988; 15: 202-5

3 DeBure C, Fiessinger J N, Priollet $P$, et al. Relationship between nailfold capillary microscopy and salivary capillary basement membrane width in Raynaud's disease and progressive systemic sclerosis. $\mathcal{f}$ Rheumatol 1985; 12: 279 82 .

4 Grassi W, Core P, Carlino G, Blasetti P, Cervini M. Labial capillary microscopy in systemic sclerosis. Ann Rheum Dis 1993; 52: 564-9.

5 Jayson M I V. Systemic sclerosis-a microvascular disorder? $f R$ Soc Med 1983; 76: 635-41.

6 LeRoy E C. A brief overview of the pathogenesis of scleroderma (systemic sclerosis). Ann Rheum Dis 1992; 51: 286-8.

7 Kahaleh M B. The molecular mechanism of endothelial cell (EC) injury in scleroderma (SSc): identification of granzyme 1 (a product of cytolytic T cell) in SSc sera [abstract]. Arthritis Rheum 1990; 67 (suppl): 33.

8 Fleischmajer R, Perlish J, Shaw K V, Pirozzi D J. Skin capillary changes in early systemic scleroderma. Electron microscopy and "in vitro" autoradiography

9 Kahaleh M B, Osborn I, LeRoy E C. Increased factor VIII von Willebrand factor antigen and von Willebrand factor activity in scleroderma and in Raynaud's phenomenon Ann Intern Med 1981; 94: 482-4.

10 Godin-Ostro E, Mitrane M, Heller I, Kim H, Seibold J R. Plasma plasminogen activator in systemic sclerosis [letter] Arthritis Rheum 1985; 4 (suppl): 28.

11 Maricq H R, Maize J C. Nailfod capillary abnormalities. Clin Rheum Dis 1982; 8: 455-78.

12 Houtman P M, Kallenberg C G M, Wouda A A, The T H. Decreased nailfold capillary density in Raynaud's phenomenon: a reflection of immunologically mediated local and systemic vascular disease? Ann Rheum Dis 1985; 44: and system

13 LeRoy E C, Smith E A, Kahaleh M B, Trojanowska M, Silver $R$ M. A strategy for determining the pathogenesis of systemic sclerosis. Is transforming growth factor $\beta$ the answer? Arthritis Rheum 1989; 32: 817-25.

14 Seibold J R. Critical tissue ischaemia in scleroderma: a note of caution. Ann Rheum Dis 1994; 53: 289-90.

15 Seibold J R. Serotonin and Raynaud's phenomenon. $f$ Cardiovasc Pharmacol 1985; 7: S95-98.

16 Bollinger A, Jäger K, Siegenthaler W. Microangiopathy of progressive systemic sclerosis: evaluation by dynamic fluoprescence videomicroscopy. Arch Intern Med 1986; 146: 1541-5.

17 Bollinger A, Jäger K, Roten A, Timeus C, Mahler F. Diffusion, pericapillary distribution and clearance of $\mathrm{Na}-$
fluorescein in the human nailfold. Pflügers Arch 1979; 382: 137-43.

18 Grassi W, Felder M, Thüring-Vollenweider U, Bollinger A. Microvascular dynamics at the nailfold in rheumatoid arthritis. Clin Exp Rheumatol 1989; 7: 47-53.
19 Grassi W, Core P, Carlino G, Cervini C. Nailfold capillary permeability in psoriatic arthritis. Scand $\mathfrak{f}$ Rheumatol 1992 21: $226-30$.

20 Mahler F, Saner H, Boss C H, Annaheim M. Local cold exposure test for capillaroscopic examination of patients with Raynaud's syndrome. Microvasc Res 1987; 33: 422-7.

21 Bollinger A, Jäger K. Trans- and pericapillary diffusion of Na-fluorescein in scleroderma and chronic venous insuffciency. Bibl Anat 1981; 20: 679-83.

22 Jayson M I V. The microcirculation in systemic sclerosis. Clin Exp Rheumatol 1984; 2: 85-91.

23 Colwell J A, Halushka P V, Sarji K E, Lopes-Virella M F Sagel J. Vascular disease in diabetes. Pathophysiological mechanisms and therapy. Arch Intern Med 1979; 139: 22530 .

24 Maricq H R, Downey J A, LeRoy E C. Standstill of nailfold blood flow during cooling in scleroderma and Raynaud's syndrome. Blood Vessels 1976; 13: 338-49.

25 Grassi W, Core P, Carlino G, Cervini C. Effects of peripheral cold exposure on microvascular dynamics in systemic sclerosis. Arthritis Rheum 1994; 37: 384-90.

26 Tsianos E V, Berecos C, Stavropoulos E, Kazakis E Danielides I C, Drosos A A. The effects of peripheral cold exposure on oesophageal motility in patients with autoimmune rheumatic diseases and Raynaud's phenomenon. Clin Rheumatol 1991; 10: 311-15.

27 Gustafsson R, Mannting F, Kazzam E, Waldenström A Hällgren $\mathbf{R}$. Cold-induced reversible myocardial ischaemia in systemic sclerosis. Lancet 1989; 2: 475-9.

28 Shuck J W, Oetgen W J, Tesar J T. Pulmonary vascular response during Raynaud's phenomenon in progressive systemic sclerosis. Am $\mathcal{f}$ Med 1985; 78: 221-7.

29 Grassi W, Core P, Carlino G, Cervini C. Acute effects of single dose nifedipine on cold-induced changes of microvascular dynamics in systemic sclerosis. $\mathrm{Br} \mathcal{F}$ Rheumatol 1994; 33: 1154-61.

30 Kahan A, Devaux J Y, Amor B, et al. Nifedipine and thallium-201 myocardial perfusion in progressive systemic thallium-201 myocardial perfusion in progress

31 Farah M J, Palmieri G M A, Sebes J I, Cremer M A, Massie $J$ D, Pinals R S. The effect of diltiazem on calcinosis in a patient with the CREST syndrome. Arthritis Rheum 1990, 33: 1287-93.

32 Duboc D, Kahan A, Maziere B, et al. The effect of nifedipine on myocardial perfusion and metabolism in systemic sclerosis. Arthritis Rheum 1991; 34: 198-203.

33 Sfikakis P P, Kyriakidis M K, Vergos C G, et al. Diffusing capacity of the lung and nifedipine in systemic sclerosis. Arthritis Rheum 1990; 33: 1634-9.

34 Rothschild B M, Masi A T. Pathogenesis of rheumatoid arthritis: a vascular hypothesis. Sem Arthritis Rheum 1982 12: $11-31$.

35 Maricq $\mathbf{H}$ R. Widefield capillary microscopy. Technique and rating scale for abnormalities seen in scleroderma and related disorders. Arthritis Rheum 1981; 24: 1159-65.

36 Duling B R. Oxygen, metabolism and microcirculatory control. In: Kaley G, Altura B M, eds. Microcirculation, Vol II. Baltimore: University Park Press, 1978.

37 Nimikoski J. Oxygen and wound healing. Clin Plastic Surg 1977; 4: 361-74.

38 Jayson M I V, Holland C D, Keegan A, Illingworth K, Taylor L. A controlled study of stanozolol in primary lor L. A controlled study of stanozolol in primary Raynaud's phenomenon and

39 Franzeck U K, Isenring G, Frey J, Bollinger A. Videodensitometric pattern recognition of $\mathrm{Na}$-fluorescein diffusion in nailfold capillary areas of patients with acrocyanosis, primary vasospastic and secondary Raynaud's phenomenon. Int Angiol 1983; 2: 143-52.

40 Ditzel J. Oxygen transport impairment in diabetes. Diabetes 1976; 25 (suppl 2): 832-8.

41 Bollinger A, Frey J, Jäger K, Furrer J, Seglias J, Siegenthaler W. Patterns of diffusion through skin capillaries in patients with long-term diabetes. N Engl $f$ Med 1982; 307: 1305-10.

42 Grassi W, Gasparini M, Cervini C. Nailfold computed videomicroscopy in morphofunctional assessment of diabetic microangiopathy. Acta Diabetol 1985; 22: 223-8.

43 Sfikakis P P, Kyriakidis M K, Vergos C G, et al. Cardiopulmonary hemodynamics in systemic sclerosis and response

44 Kahan A, Amor B, Menkes C J, Weber S. Nifedipine in digital ulceration in scleroderma [letter]. Arthritis Rheum 1983; 26: 809 .

45 Jaffe I A. Nifedipine in digital ulcerations in scleroderma [letter]. Arthritis Rheum 1982; 25: 1267-8.

46 Goodfield M J D, Rowell N R. Hand warming as a treatment for Raynaud's phenomenon in systemic sclerotreatment for Raynaud's phenomen.
sis. Br $\mathcal{}$ Dermatol 1988; 119: 643-6.

47 Cooke E D, Nicolaides A N. Raynaud's syndrome. BMF 1990; 300: 553-5.

48 Medsger T A Jr. Treatment of systemic sclerosis. Ann Rheum Dis 1991 ; 50: 877-86. 